\title{
Bridge Dynamic Displacement Monitoring Using Geodetic Measurement
}

\author{
Alexandra-Nadia CÎRDEI ${ }^{1^{*}}$, Dumitru ONOSE ${ }^{1}$ \\ ${ }^{1}$ Faculty of Geodesy, Tehnical University of Civil Engeneering Bucharest, Romania \\ *corresponding author: c.alexandranadia@gmail.com
}

BulletinUASVM Horticulture 76(1) / 2019

Print ISSN 1843-5254, Electronic ISSN 1843-5394

DOI:10.15835/buasvmcn-hort: 2018.0027

\begin{abstract}
The paper analyzes the possibility to define a dynamic model associated to a bridge using the results of geodetic measurements made for displacement monitoring using modern calculation models. Combining the results obtained with two modern calculation models (Finite Element Method and Kalman Filter) we can define a complex model, able of meeting current risk management requirements. The paper aims to highlight the importance and usefulness of realizing the dynamic model of a bridge in order to test hypotheses regarding limit situations such as: testing the structure response in case of a flood or in the event of a major earthquake, determining the maximum admissible weight on the bridge without requiring interventions, determining the areas where the bridge requires consolidation work after a certain period of time or after natural phenomena etc.
\end{abstract}

Keywords: bridge, displacement, monitoring, prediction

\section{Introduction}

Analyzing the way an important structure will behave in the future, especially taking into consideration exceptional situation it might face, is a very important stage in the context in which protection of people and the environment has become a priority for all developed or developing contries.

Bridges are part of the major construction category making it easier to get from one place to another, especially in mountain areas, those crossed by rivers or those separated by valleys.

The way of building the bridges, as well as the materials used for their construction, evolved simultaneously with human civilization, and it has been necessary to be permanently adapted to the human lifestyle and subsequently to the means of transport used by them.

Bridges and other artworks show the progress and degree of development of a contry.

There are many bridges that have been built a long time ago, to ensure the continuity of a terrestrial communication path over a natural or artificial barrier, that continues to be used by people today, but to ensure that their exploitation does not endanger the people or the environment, it is necessary to analyse their behaviour and carry out maintenance work.

By destination, bridges can be classified as: road, rail, aqeducte, combined etc. In this paper we will refer to a road bridge, because their importance has increased due to the steady growth in the number of vehicles. This trend was imposed by the development of national and international road networks resulting from the development of urban areas and the need to streamline traffic in the intensely circulated areas, especially those that ensure entry and exit from cities.

In our country the road bridges have known a pronounced development after 1953 when IPTANA (Institute for Shipping, Naval and Air Transport Design) was established.

The construction and execution of maintenance works for such a complex structure involves 
the realization of a complex project, combining several areas of activity and at the same time taking into consideration both the technical and the economic aspect.

Monitoring the bridge displacements specific in the exploitation stage is particularly important as it consists in observing and recording some aspects, phenomena and parameters that may indicate changes in the construction's capacity to meet the resistance, stability and sustainability requirements established by the project.

Starting from the tracking results, a deformation model specific to the studied structure can be defined, which can then be stabilized in order to predict the most likely values of the displacements, taking into account normal operating conditions and lack of extreme phenomena.

Following the realization of the dynamic model of the bridge combining Extended Kalman Filter results, for the prediction of displacemnts for stages 3,4,5 and 6, with the Finite Element Method, for defining the model itself, the displacements analysis it will offer the possibility to perform different tests, regarding various hypotheses. In recent years, road traffic has intensified and the trend for this process is to continue, so the analysis of how the bridge is able to support this process is particularly important and it is one of the main uses for a model thus defined.

\section{Materials and methods}

The studied bridge is built over the DanubeBlack Sea Canal and is located on the A2 highway connecting Bucharest and Constanta. It consists of two independent structures made of the same material, symmetrical to the highway axis, each of them serving a direction. Both bridges have a continuous beam structure made by console molding, having three openings.

The superstructure of the bridge is supported by seismic supports on the two-pile and two abutments infrastructure. The two piles have the same shape, but different heights, respectively $16,15 \mathrm{~m}$ and $17,40 \mathrm{~m}$, and the two abutments are made identical, drowned type.

In order to achieve the dynamic model of the bridge, it is necessary to have information on several measurement steps obtained with geometric leveling for the 39 marks considered at the characteristic points of the bridge, both on the marginal and in the central area. Measurements have been made with a leveling instrument, digital level Leica Sprinter 200M, characterized by a precision of $1.5 \mathrm{~mm}$ per double kilometer of levelment. The marks are suggestively represented in Figure 1 in the two-dimensional space (X and $\mathrm{Y}$ coordinates).

The first two measurement stages made during the flow traffic will be used for the deformation analysis, and the displacements size will be determined over a reference stage (stage 0 ) made under the same conditions, after the bridge was put into use. Starting from the results obtained in the two steps of the deformation tracking measurements (stages 1 and 2), the Extended Kalman Filter is used to obtain predictions of the results to be obtained at the next four steps (stages 3,4,5 and 6) for the construction taken into consideration, considering the same reference stage results since several steps of measurement are required to create and stabilize the deformation model (Tab. 1.).

The Kalman filter is a particularly powerful mathematical tool used very often in modern computer systems to represent the real world as Mohinder and Angus (2014) have demonstrated with many examples in their book. Implementing this method in the form of an algorithm makes it very easy to use, especially in the context of continuous development of computing techniques, replacing analogue estimation methods.

A great advantage of this method is that it is compatible with formulating and solving the characteristic states of dynamic systems. The difficulty or the impossibility of accurately shaping system noise may be a limitation of the application of the method.

The discrete Kalman filter is the initially formulated solution and refers to the use of the results of the measurements made to estimate the state in discrete points over time. The extended Kalman filter is the solution that later emerged from the need to solve and analyze processes that can not be translated into linear differential equations.

The results of using this method can be divided into two categories, namely: time-lapse equations, which can be regarded as prediction equations of the analyzed phenomenon, and measurement update equations, which can be regarded as equations for correcting the results of the measurements. 


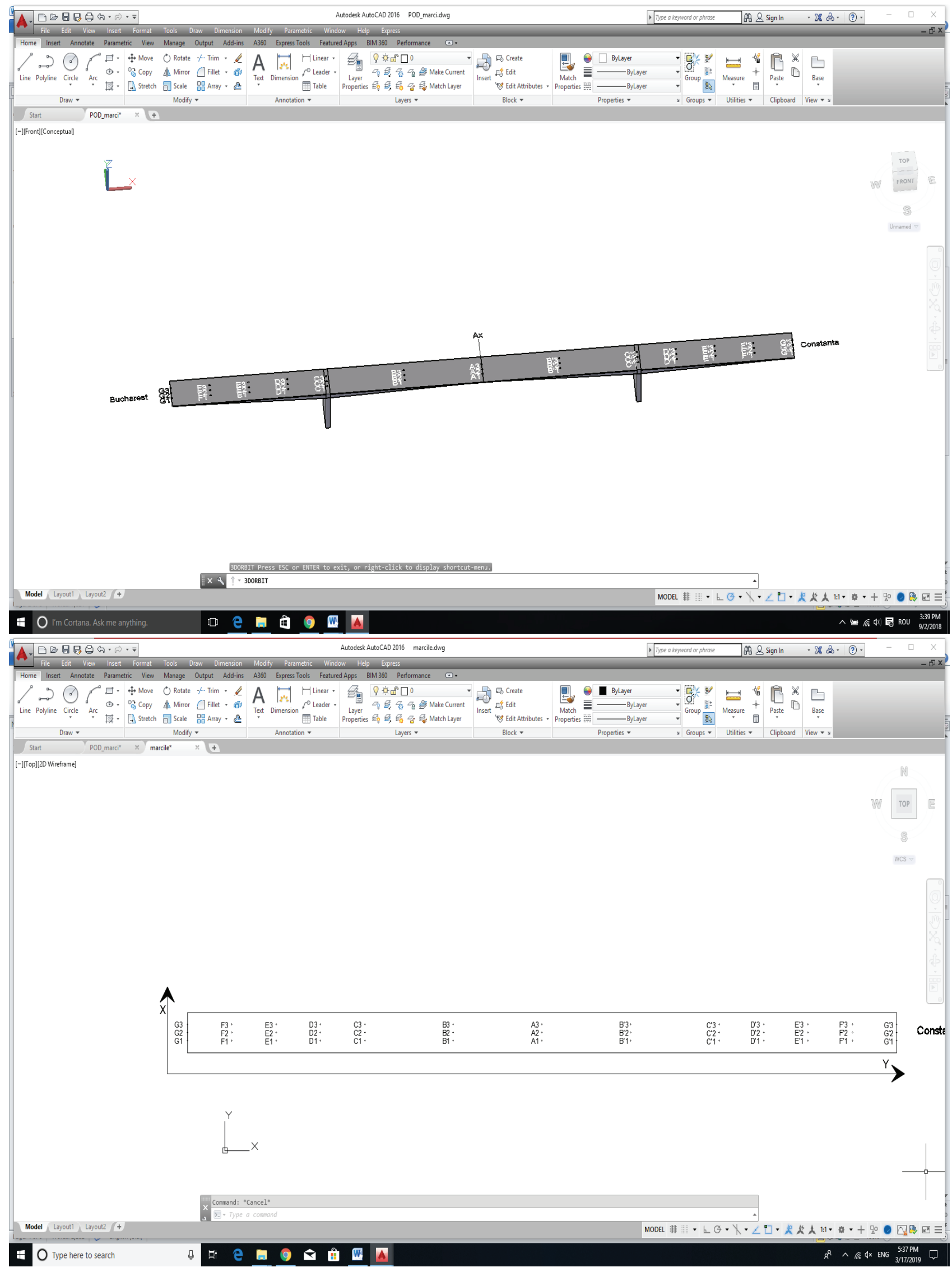

Figure 1. Layout of tracking marks 
Table 1. Displacement results

\begin{tabular}{|c|c|c|c|c|c|c|c|}
\hline Mark & $\mathrm{H}_{0}[\mathrm{~m}]$ & $\Delta \mathbf{h}_{0-1}[\mathrm{~mm}]$ & $\Delta \mathbf{h}_{0-2}[\mathrm{~mm}]$ & $\Delta \mathbf{h}_{0-3}{ }^{*}[\mathrm{~mm}]$ & $\Delta \mathbf{h}_{\mathbf{0 - 4}}{ }^{*}[\mathrm{~mm}]$ & $\Delta \mathbf{h}_{0-5}{ }^{*}[\mathbf{m m}]$ & $\Delta \mathbf{h}_{0-6}{ }^{*}[\mathrm{~mm}]$ \\
\hline G'1 & 50.0126 & 0.3 & 0.1 & 0.2 & 0.2 & 0.4 & 0.1 \\
\hline$G^{\prime} 2$ & 50.0538 & 0.3 & 0.0 & 0.2 & 0.2 & 0.4 & 0.2 \\
\hline$G^{\prime} 3$ & 50.3096 & 0.3 & 0.1 & 0.2 & 0.2 & 0.3 & 0.0 \\
\hline$F^{\prime} 1$ & 50.0875 & 1.8 & 0.1 & 0.1 & 0.2 & -1.3 & 0.2 \\
\hline$F^{\prime} 2$ & 50.1373 & 1.7 & 0.0 & 0.0 & 0.2 & -1.4 & 0.2 \\
\hline$F^{\prime} 3$ & 50.3920 & 1.8 & 0.1 & 0.0 & 0.2 & -1.4 & 0.1 \\
\hline E'1 & 50.2257 & 2.1 & 0.1 & 0.0 & 0.2 & -1.3 & 0.1 \\
\hline$E^{\prime} 2$ & 50.2843 & 1.9 & 0.0 & -0.1 & 0.1 & -1.5 & 0.2 \\
\hline E'3 & 50.5363 & 2.0 & 0.1 & -0.1 & 0.1 & -1.5 & 0.1 \\
\hline D'1 & 50.3671 & 1.4 & 0.1 & 0.1 & 0.2 & -0.5 & 0.2 \\
\hline$D^{\prime} 2$ & 50.4301 & 1.3 & 0.0 & 0.0 & 0.2 & -0.6 & 0.2 \\
\hline$D^{\prime} 3$ & 50.6826 & 1.3 & 0.0 & 0.0 & 0.1 & -0.7 & 0.0 \\
\hline$C^{\prime} 1$ & 50.5109 & 0.3 & 0.1 & 0.2 & 0.1 & 0.3 & 0.1 \\
\hline$C^{\prime} 2$ & 50.5753 & 0.2 & 0.0 & 0.1 & 0.1 & 0.2 & 0.2 \\
\hline$C^{\prime} 3$ & 50.8144 & 0.2 & 0.0 & 0.1 & 0.0 & 0.2 & 0.0 \\
\hline B'1 & 50.7880 & -2.7 & -0.1 & 0.5 & -0.1 & 1.5 & -0.1 \\
\hline$B^{\prime} 2$ & 50.8465 & -2.7 & -0.1 & 0.5 & -0.1 & 1.4 & -0.1 \\
\hline$B^{\prime} 3$ & 51.0907 & -2.7 & -0.1 & 0.5 & -0.1 & 1.4 & -0.3 \\
\hline A1 & 51.0801 & -2.6 & -0.2 & 1.4 & -0.4 & 1.1 & -0.5 \\
\hline A2 & 51.1281 & -2.6 & -0.3 & 1.3 & -0.4 & 1.0 & -0.5 \\
\hline A3 & 51.3765 & -2.6 & -0.2 & 1.3 & -0.4 & 1.0 & -0.6 \\
\hline B1 & 51.3526 & -2.7 & -0.1 & 1.6 & -0.1 & 0.3 & -0.3 \\
\hline B2 & 51.4050 & -2.7 & -0.1 & 1.5 & 0.0 & 0.4 & -0.1 \\
\hline B3 & 51.6566 & -2.7 & -0.1 & 1.5 & -0.2 & 0.2 & -0.4 \\
\hline C1 & 51.6248 & 0.2 & 0.0 & 0.2 & -0.1 & 0.1 & 0.0 \\
\hline C2 & 51.6745 & 0.2 & 0.0 & 0.1 & 0.1 & 0.2 & 0.2 \\
\hline C3 & 51.9229 & 0.3 & 0.0 & 0.1 & -0.1 & 0.0 & -0.1 \\
\hline D1 & 51.7475 & 1.2 & 0.0 & -0.8 & 0.0 & -0.1 & 0.1 \\
\hline D2 & 51.8048 & 1.3 & 0.0 & -0.8 & 0.1 & 0.1 & 0.2 \\
\hline D3 & 52.0568 & 1.3 & 0.0 & -0.8 & -0.1 & -0.1 & -0.1 \\
\hline E1 & 51.8780 & 1.9 & 0.0 & -1.6 & -0.1 & -0.2 & 0.0 \\
\hline E2 & 51.9194 & 1.9 & 0.0 & -1.7 & 0.0 & -0.1 & 0.2 \\
\hline E3 & 52.1766 & 2.0 & 0.0 & -1.7 & -0.1 & -0.2 & -0.1 \\
\hline F1 & 52.0004 & 1.7 & 0.0 & -1.6 & 0.0 & -0.2 & 0.1 \\
\hline F2 & 52.0457 & 1.7 & 0.0 & -1.7 & -0.1 & -0.2 & 0.1 \\
\hline F3 & 52.3048 & 1.7 & 0.1 & -1.6 & 0.0 & -0.1 & 0.0 \\
\hline G1 & 52.1343 & 0.2 & 0.0 & 0.1 & -0.1 & 0.0 & 0.0 \\
\hline G2 & 52.1950 & 0.2 & 0.0 & 0.0 & -0.1 & 0.0 & 0.0 \\
\hline G3 & 52.4319 & 0.2 & 0.0 & 0.1 & -0.1 & -0.1 & -0.1 \\
\hline
\end{tabular}

*-predicted results

To apply the method, the Matlab R2018a software has been used, allowing the use of the Kalman filter both discrete and extended.
Based on the measurements and predictions results obtained, the deformation model will be created and stabilized. Taking into consideration 
that we have the results of geometric leveling measurements also for the stages 3 and 4, with the first four steps results we can create and validate the model. Then with the last two stages results predicted using Extended Kalman Filter the model is stabilized using the ANSYS software based on the finite element method, as detailed by Moaveni (1999).

The Finite Element Method was designed to be used with computers to solve differential equations associated with a physical problem for complicated geometries. Also, this method allows the realization of a mathematical calculation model associated with the real system, much easier and economically to be modified and adjusted than a prototype.

Currently, the Finite Element Method has become one of the most used and powerful tools used to solve engineering problems. Lateș (2008) presents the Finite Element Analysis of a resistance structure model as a numerical verification for a structure that has a well defined geometry, a given load and well-defined bearing conditions. In this way the values of the displacements, stresses and reactions in the bearing, the vibration frequencies own, etc can be obtained.

A great advantage of the method is that it offers the possibility of modeling non-homogeneous bodies in terms of physical properties and has a high degree of generality. The method is, however, an approximate one, and the results are difficult to satisfy with high precision requirements.

The computational program used to analyze the problem is applied to a model of the actual structure studied by a user who approximates the model as best as possible, taking into account its main characteristics. The process of building this model is called modeling. In this paper we will use an ideal model, formulated in mathematical terms and notions.

\section{Results and discussions}

Displacement values determined by the Extended Kalman Filter method have associated noise values, that must be filtered before being used in the Finite Element Method. For this, we will use the results of the predictions for steps 3 and 4 and the results for the same steps obtained from the

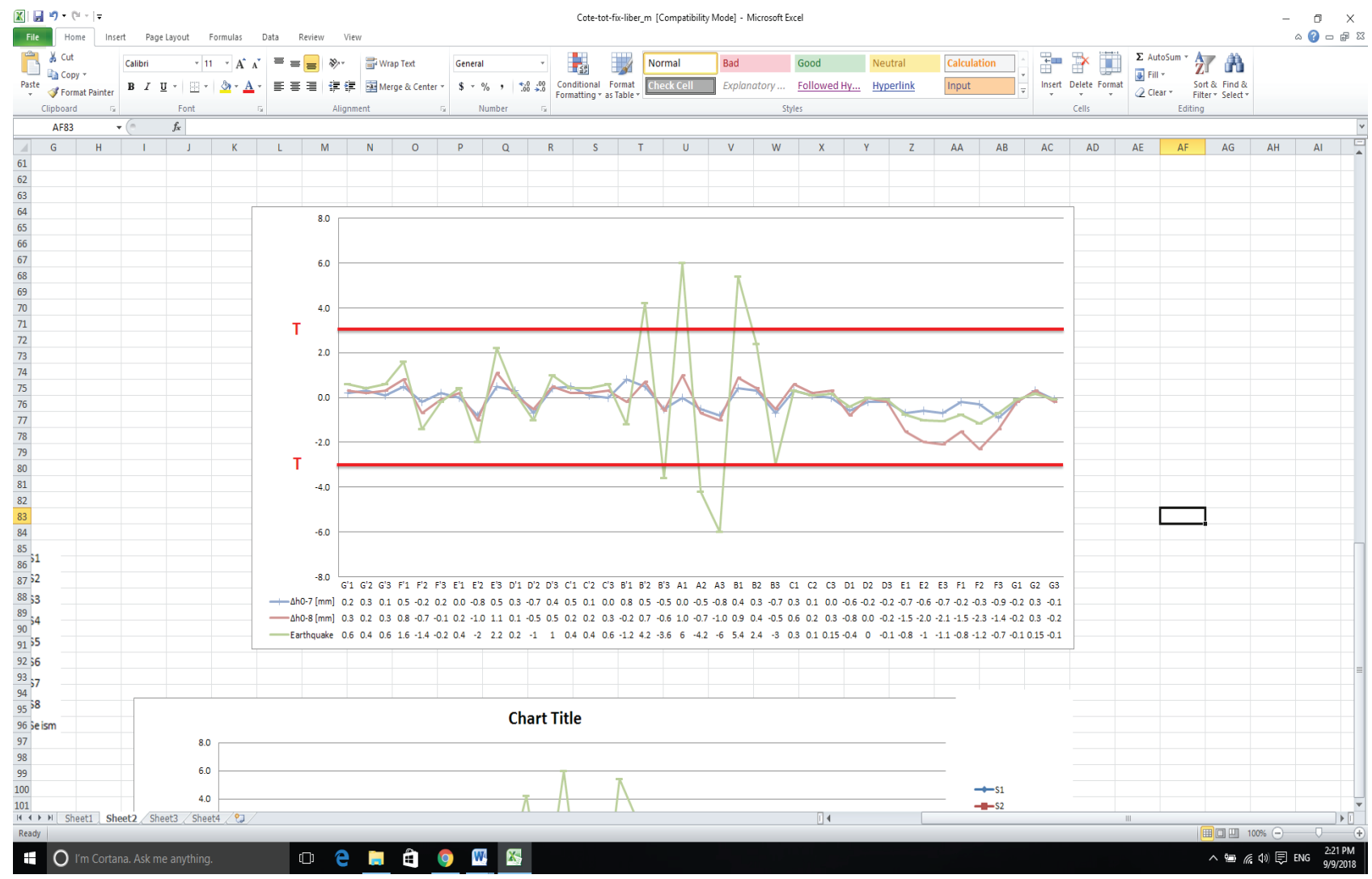

Figure 2. Prediction of bridge behaviour 
measurements. The resulting noise will be further elucidated from the results obtained with the Extended Kalman Filter, and then it can be used to create and stabilize the deformation model.

The resulting dynamic model can be used to test different situations and to make predictions about structure behavior in the event that the bridge would undergo exceptional situations.

The results obtained with this model consist in the size of the deformations we can expect, in the direction of elevation coordinate $(\mathrm{H})$ for the 39 tracked marks with an average accuracy of \pm 0.3 $\mathrm{mm}$.

According to the results obtained using the dynamic model for stages 7 and 8 over the same reference stage and taking into consideration normal operating conditions and without the occurrence of extreme phenomena, it can be seen that for the next two years the structure can support traffic if it maintains or diminishes its speed of amplification.

Also following the results obtained after a test carried out on the same model, amplifying the vibrations and taking into considerations results obtained by Liu et al. (2009) and Yang et al (2005) for a similar case, it can be seen that if it would be subjected to vibrations of a major earthquake, with a magnitude greater than 6 on the Richter scale, the bridge would require maintenance work in the central area.

\section{Conclusion}

The importance of making predictions about the way bridges will behave is particularly important, especially because there are real recent examples, at national and international level, demonstrating the magnitude of the negative impact of the total or partial deterioration of such structures.
In addition to the information provided by the structure's behavioral tracking over time, the results obtained with a deformation model can be used to make predictions about the behavior of the structure and allow the study of various limit situations, associated with some exceptional natural phenomena that can not be controlled, on the created digital model. By analyzing these results, precautions can be taken to prevent them, so that the ability of the structure to provide safety to people and the environment is not affected.

Using the deformation model created and stabilized, it will be possible to determine the maximum traffic flow that the bridge can sustain without affecting the structure. It is also possible to determine the places where it should undergo interventions so that the structure can be safely exploited without threathning humans and the environment, even in the context of increased traffic. This research did not receive any specific grant from funding agencies in the public, commercial, or not-for-profit sectors.

\section{References}

1. Lateș M.T. (2008). Metoda Elementelor Finite, Transilvania University Publishing House, Brașov, ISBN 978-973-635-659-9

2. Liu X., Escamilla-Ambrosio P.J., Lieven N.A.J. (2009) Extended Kalman filtering for the detection of damage in linear mechanical structures, Journal of Sound and Vibration, 325:1023-1046

3. Moaveni S. (1999) Finite Element Analysis, Theory and Application with ANSYS, Pretince Hall, New Jersey

4. Mohinder G., Angus A. (2014) Kalman Filtering: Theory and Practice with MATLAB 4th Edition, John Wiley \& Sons, Inc., ISBN 978-1-118-85121-0

5. Yang J.N., Lin S., Huang H., Zhou L. (2005) An adaptive extended Kalman filter for structural damage identification, Journal of Structual Control Health Monitoring, 13:849-867 